\title{
Average Damage Caused by Multiple Weapons against an Area Target of Normally Distributed Elements
}

\author{
Hongyun Wang1, George Labaria' ${ }^{1}$ Cardy Moten ${ }^{2}$, Hong Zhou ${ }^{3 *}$ \\ ${ }^{1}$ Department of Applied Mathematics and Statistics University of California, Santa Cruz, CA, USA \\ ${ }^{2}$ TRADOC Analysis Center Naval Postgraduate School, Monterey, CA, USA \\ ${ }^{3}$ Department of Applied Mathematics Naval Postgraduate School, Monterey, CA, USA \\ Email: ${ }^{* h z h o u @ n p s . e d u ~}$
}

How to cite this paper: Wang, H.Y., Labaria, G., Moten, C. and Zhou, H. (2017) Average Damage Caused by Multiple Weapons against an Area Target of Normally Distributed Elements. American Journal of $O p$ erations Research, 7, 289-306.

https://doi.org/10.4236/ajor.2017.75022

Received: July 4, 2017

Accepted: September 12, 2017

Published: September 15, 2017

Copyright $\odot 2017$ by authors and Scientific Research Publishing Inc. This work is licensed under the Creative Commons Attribution International License (CC BY 4.0).

http://creativecommons.org/licenses/by/4.0/

\begin{abstract}
This paper investigates the effect of launching multiple weapons against an area target of normally distributed elements. We provide an analytical form of the average damage fraction and then apply it to obtain optimal aimpoints. To facilitate the computational efforts in practice, we also consider optimizations over given constrained patterns of aimpoints. Finally, we derive scaling laws for optimal aimpoints and optimal damage fraction with respect to the radius of the area target.
\end{abstract}

\section{Keywords}

Area Target, Carleton Damage Function, Average Damage Fraction, Optimal Aimpoints, Scaling Laws

\section{Introduction}

The theory of firing, which mainly concerns aiming, kill probability and allocation of munitions, was inspired by World War II and has been progressed significantly in the past decades [1]. A brief history of firing theory can be found in Washburn and Kress's book [2] where the authors also presented a detailed discussion on shooting without feedback or with feedback. Another good reference on weaponeering is given by Driels [3].

In this paper we are interested in studying the effect of precision-guided munitions such as Excaliburs. These coordinate-seeking munitions are usually guided by radio, radar, or laser and launched by a cannon. They are intended to hit a target accurately and cause minimal collateral damage to civilians, friendly 
forces and infrastructure, especially hospitals, schools, churches, and residential homes. The precision-guided weapons are in general subject to target-location errors and ballistic dispersion errors. The target-location errors, or aiming errors, result from inaccuracies associated with identifying a target's location. In contrast, the ballistic dispersion errors are caused by random weapons effects, which may vary from one weapon to another and are assumed to be independent from shot to shot. When a single weapon is fired, it is natural to aim it at the expected center of the target. However, when multiple weapons are launched against a unitary target, the probability of damaging the target can be improved significantly by spreading the aimpoints around the target and the optimal distribution of aimpoints has been investigated in [4] and [5]. Our goal here is to extend our previous studies to estimate the probability of destroying an area target of normally distributed elements with multiple weapons. We will seek optimal aimpoints for various number of weapons.

The plan of this paper is to first review our previous analytical results for the case of multiple weapons against a single target in Section 2. Section 3 introduces the mathematical problem of multiple weapons being released against an area target consisting of normally distributed elements. Exact solution for the average damage fraction is then derived. Section 4 calculates the optimal aiming points and examines the relation among the radius of area target, the number of weapons and the optimal (maximum) damage fraction. In addition to the unconstrained overall optimization of the damage fraction, we also study empirical, fast and robust constrained optimization over several prescribed patterns. The goal is to reduce the computational complexity of optimization and to compute a set of nearly optimal aimpoints efficiently. Section 5 provides scaling laws for optimal aimpoints and optimal damage fraction with respect to the radius of area target. Section 6 highlights conclusions.

\section{Review of Our Previous Analytical Results for the Case of Multiple Weapons against a Single Target}

Even though the world is three-dimensional, most targets are known to be on the surface of the Earth and therefore the targets are assumed to be in a two-dimensional ground space. Conventionally, we use two coordinates to define this ground plane: the range direction and the deflection direction. The range direction is defined by the direction of the weapon's velocity vector, whereas the deflection direction is perpendicular to the range direction.

Previously [5] we have studied the case of multiple weapons with both dependent and independent errors against a single target positioned at $\boldsymbol{x}_{\text {target }}=$ $(0,0)$. For reader's convenience, we review briefly the mathematical formulation of the problem. Let

- $\boldsymbol{r}_{j}=$ the aiming point of weapon $j$ for $j=1,2, \cdots, M$.

- $\boldsymbol{Y}=$ the dependent error of $M$ weapons, affecting the impact points of all $M$ weapons uniformly. For example, $\boldsymbol{Y}$ is the part of error associated with 
identifying the target location incorrectly/inaccurately. We assume $\boldsymbol{Y}$ is a normal random variable.

- $\quad \boldsymbol{X}_{j}=$ independent error of weapon $j$, affecting only the impact point of weapon $j$ individually. For example, $\boldsymbol{X}_{j}$ is the part of error associated with aiming and firing weapon $j$. We assume that $\left\{\boldsymbol{X}_{j}, j=1,2, \cdots, M\right\}$ are normal random variables, independent of each other and independent of normal random variable $\boldsymbol{Y}$.

We model the dependent error $\boldsymbol{Y}$ as a normal random variable with zero mean:

$$
\boldsymbol{Y} \sim N\left(\left(\begin{array}{l}
0 \\
0
\end{array}\right),\left(\begin{array}{cc}
\sigma_{1}^{2} & 0 \\
0 & \sigma_{2}^{2}
\end{array}\right)\right)
$$

where $\sigma_{1}$ and $\sigma_{2}$ are standard deviations, respectively, in the range and the deflection directions, which give an indication of the spread of the dependent error in these two directions. We model each independent error $\boldsymbol{X}_{j}$ as a normal random variable with zero mean:

$$
\boldsymbol{X}_{j} \sim N\left(\left(\begin{array}{l}
0 \\
0
\end{array}\right),\left(\begin{array}{cc}
d_{1}^{2} & 0 \\
0 & d_{2}^{2}
\end{array}\right)\right)
$$

The impact point of weapon $j$ is given by

$$
\boldsymbol{w}_{j}=\boldsymbol{r}_{j}+\boldsymbol{Y}+\boldsymbol{X}_{j}
$$

We use the Carleton damage function described below to model the probability of killing by an individual weapon. Let $\boldsymbol{w}=(w(1), w(2))$ be the impact point of a weapon where $w(1)$ and $w(2)$ denote respectively the range component and the deflection component of the impact point from the target. In Carleton damage function, the probability of the target being killed by a weapon at impact point $\boldsymbol{w}$ is mathematically modeled as

$$
\begin{aligned}
& \operatorname{Pr}(\text { target being killed by one weapon at impact point } \boldsymbol{w}) \\
& =\exp \left(\frac{-w(1)^{2}}{2 b_{1}^{2}}\right) \exp \left(\frac{-w(2)^{2}}{2 b_{2}^{2}}\right)
\end{aligned}
$$

This is the well-known Carleton damage function or the diffuse Gaussian damage function [2]. The two parameters $b_{1}$ and $b_{2}$ in the Carleton damage function (1) represent the effective weapon radii in the range and deflection directions, respectively.

The probability of a target being killed by the $M$ weapons, averaged over all random errors (i.e., dependent and independent errors), is called the kill probability and is mathematically denoted by $p_{\text {kill }}$ (target, $M$ weapons). Note that in the notation for the kill probability, the target identity is explicitly included. This will be very convenient later in the discussion of an area target with multiple target elements, in which we can study the kill probability for each individual element.

With the impact points of the $M$ weapons given by the random variable 
$\left\{\boldsymbol{w}_{j}=\boldsymbol{r}_{j}+\boldsymbol{Y}+\boldsymbol{X}_{j}, j=1,2, \cdots, M\right\}$, we derived an analytical expression for the kill probability (averaged over the distribution of impact points) as a function of quantities

$$
\begin{gathered}
\left(\sigma_{1}, \sigma_{2}, d_{1}, d_{2}, b_{1}, b_{2},\left\{\boldsymbol{r}_{j}, j=1,2, \cdots, M\right\}\right) . \\
p_{\text {kill }}(\text { target, } M \text { weapons })=G\left(\sigma_{1}, \sigma_{2}, d_{1}, d_{2}, b_{1}, b_{2},\left\{\boldsymbol{r}_{j}, j=1,2, \cdots, M\right\}\right)
\end{gathered}
$$

where function $G$ is defined as

$$
\begin{aligned}
G & \left(\sigma_{1}, \sigma_{2}, d_{1}, d_{2}, b_{1}, b_{2},\left\{\boldsymbol{r}_{j}, j=1,2, \cdots, M\right\}\right) \\
= & -\sum_{k=1}^{M}(-1)^{k} \sum_{\left(j_{1}, \cdots, j_{k}\right)} E\left[F_{1}\left(j_{1}, \cdots, j_{k}\right)\right] E\left[F_{2}\left(j_{1}, \cdots, j_{k}\right)\right] \\
E\left[F_{1}\left(j_{1}, \cdots, j_{k}\right)\right]= & \left(\frac{b_{1}^{2}}{b_{1}^{2}+d_{1}^{2}}\right)^{\frac{k}{2}}\left(\frac{\left(b_{1}^{2}+d_{1}^{2}\right) / k}{\left(b_{1}^{2}+d_{1}^{2}\right) / k+\sigma_{1}^{2}}\right)^{\frac{1}{2}} \\
& \times \exp \left(\frac{\left(\sum_{l=1}^{k} r_{j_{l}}(1) / k\right)^{2}-\sum_{l=1}^{k} r_{j_{l}}(1)^{2} / k}{2\left(b_{1}^{2}+d_{1}^{2}\right) / k}-\frac{\left(\sum_{l=1}^{k} r_{j_{l}}(1) / k\right)^{2}}{2\left(\left(b_{1}^{2}+d_{1}^{2}\right) / k+\sigma_{1}^{2}\right)}\right) \\
E\left[F_{2}\left(j_{1}, \cdots, j_{k}\right)\right]= & \left.\left(\frac{b_{2}^{2}}{b_{2}^{2}+d_{2}^{2}}\right)^{\frac{k}{2}}\left(\frac{\left(b_{2}^{2}+d_{2}^{2}\right) / k}{\left(b_{2}^{2}+d_{2}^{2}\right) / k+\sigma_{2}^{2}}\right)^{\frac{1}{2}}\right) \\
& \times \exp \left(\frac{\left(\sum_{l=1}^{k} r_{j_{l}}(2) / k\right)^{2}-\sum_{l=1}^{k} r_{j_{l}}(2)^{2} / k}{2\left(b_{2}^{2}+d_{2}^{2}\right) / k}-\frac{\left(\sum_{l=1}^{k} r_{j_{l}}(2) / k\right)^{2}}{2\left(\left(b_{2}^{2}+d_{2}^{2}\right) / k+\sigma_{2}^{2}\right)}\right)
\end{aligned}
$$

Mathematically, $F_{1}\left(j_{1}, \cdots, j_{k}\right)$ is the product, over $k$ weapons $\left\{j_{1}, \cdots, j_{k}\right\}$, of all factors involving only components in the range direction (i.e., $\sigma_{1}, d_{1}, b_{1}$, $r(1))$ while $F_{2}\left(j_{1}, \cdots, j_{k}\right)$ is the product, over $k$ weapons $\left\{j_{1}, \cdots, j_{k}\right\}$, of all factors involving only components in the deflection direction (i.e., $\sigma_{2}, d_{2}, b_{2}$, $r(2)$ ). Together, Equations (2), (3), (4) and (5) form an explicit analytical expression for the kill probability of a point target, $p_{\text {kill }}$ (target, $M$ weapons). This analytical solution will be used in next section to calculate the damage fraction of an area target consisting of normally distributed target elements.

\section{Mathematical Formulation: Multiple Weapons against an Area Target of Normally Distributed Elements}

Now let us examine the situation where $M$ weapons are used against an area target centered at $\boldsymbol{x}_{\text {target }}=(0,0)$, consisting of $N$ discrete elements, normally distributed around the center. Let

- $\boldsymbol{Z}_{k}=$ the location of element $k$ of the area target, for $k=1,2, \cdots, N$.

- $\boldsymbol{r}_{j}, \boldsymbol{X}_{j}$, and $\boldsymbol{Y}$ are the same as defined in Section 2. They are respectively, the aiming point, the independent error, and the dependent error of weapon 
$j$.

In this situation, $\boldsymbol{Z}_{k}$, the location of element $k$ of the area target, is modeled as a normal random variable with zero mean:

$$
Z_{k} \sim N\left(\left(\begin{array}{l}
0 \\
0
\end{array}\right),\left(\begin{array}{cc}
s_{1}^{2} & 0 \\
0 & s_{2}^{2}
\end{array}\right)\right)
$$

We assume that $\left\{\boldsymbol{Z}_{k}, k=1,2, \cdots, N\right\}$ are independent of each other, and are independent of $\boldsymbol{X}_{j}$ and $\boldsymbol{Y}$.

Figure 1 shows a sample distribution for an area target of 20 elements normally distributed with $s_{1}=s_{2}=4$.

To study the damage fraction caused by the $M$ weapons on the area target, we examine the kill probability of element $k$ caused by the $M$ weapons. The impact point of weapon $j$ relative to element $k$ of the area target is given by

$$
\boldsymbol{w}_{j}^{(k)}=\boldsymbol{r}_{j}+\boldsymbol{Y}+\boldsymbol{X}_{j}-\boldsymbol{Z}_{k} \equiv \boldsymbol{r}_{j}+\boldsymbol{Y}^{(k, \text { eff })}+\boldsymbol{X}_{j}
$$

where the effective dependent error of the $M$ weapons relative to element $k$ is defined as

$$
\boldsymbol{Y}^{(k, \text { eff })} \equiv \boldsymbol{Y}-\boldsymbol{Z}_{k}
$$

Note that $\boldsymbol{Y}^{(k, \text { eff })}$ is a normal random variable with zero mean

$$
\boldsymbol{Y}^{(k, \text { eff })} \sim N\left(\left(\begin{array}{l}
0 \\
0
\end{array}\right),\left(\begin{array}{cc}
\sigma_{1}^{2}+s_{1}^{2} & 0 \\
0 & \sigma_{2}^{2}+s_{2}^{2}
\end{array}\right)\right)
$$

The kill probability of element $k$ caused by the $M$ weapons (averaged over random independent errors $\left\{\boldsymbol{X}_{j}, j=1,2, \cdots, M\right\}$, over the random dependent error $\boldsymbol{Y}$, and over the random element location $\boldsymbol{Z}_{k}$ ) is given by

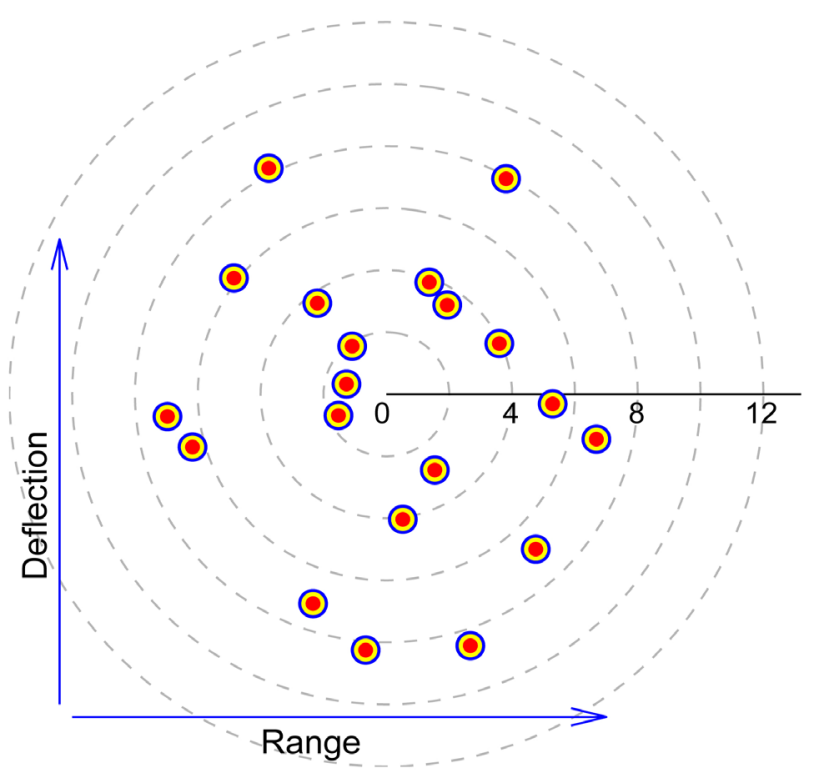

Figure 1. A sample distribution for an area target consisting of 20 random elements normally distributed with $s_{1}=s_{2}=4$, as described by (6). 


$$
\begin{aligned}
& p_{\text {kill }}(\text { element } k, M \text { weapons }) \\
& =G\left(\sqrt{\sigma_{1}^{2}+s_{1}^{2}}, \sqrt{\sigma_{2}^{2}+s_{2}^{2}}, d_{1}, d_{2}, b_{1}, b_{2},\left\{\boldsymbol{r}_{j}, j=1,2, \cdots, M\right\}\right)
\end{aligned}
$$

where function $G$ is defined in Equations (3), (4) and (5). Notice that in the case of an area target of normally distributed elements, the kill probability of element $k$ has exactly the same form as in the case of a single target at $(0,0)$ with the exception that all instances of $\sigma_{1}^{2}$ be replaced by $\left(\sigma_{1}^{2}+s_{1}^{2}\right)$ and $\sigma_{2}^{2}$ be replaced by $\left(\sigma_{2}^{2}+s_{2}^{2}\right)$.

Let $\chi_{k}$ be the Bernoulli random variable indicating whether or not element $k$ is killed (" 1 " corresponding to "killed"). The damage fraction (random variable) of the area target is the number of elements killed normalized by the total number of elements.

$$
q_{\text {damage }}(\text { area target, } M \text { weapons }) \equiv \frac{1}{N} \sum_{k=1}^{N} \chi_{k}
$$

The average damage fraction has the expression

$$
\begin{aligned}
& E\left[q_{\text {damage }}(\text { area target, } M \text { weapons })\right] \\
& =E\left[\frac{1}{N} \sum_{k=1}^{N} \chi_{k}\right]=\frac{1}{N} \sum_{k=1}^{N} p_{\text {kill }}(\text { element } k, M \text { weapons }) \\
& =G\left(\sqrt{\sigma_{1}^{2}+s_{1}^{2}}, \sqrt{\sigma_{2}^{2}+s_{2}^{2}}, d_{1}, d_{2}, b_{1}, b_{2},\left\{\boldsymbol{r}_{j}, j=1,2, \cdots, M\right\}\right)
\end{aligned}
$$

Expression (9) gives the exact solution for the case of an area target of normally distributed elements.

When both the independent error $\left(\boldsymbol{X}_{j}, j=1,2, \cdots, M\right)$ and the dependent error $(\boldsymbol{Y})$ of the $M$ weapons are absent, random variables $\left\{\chi_{k}, k=1,2, \cdots, N\right\}$ are independent of each other. In this situation, we can calculate analytically the standard deviation of damage fraction (random variable). The variance of damage fraction has the expression

$$
\begin{aligned}
& \operatorname{var}\left[q_{\text {damage }}(\text { area target, } M \text { weapons })\right] \\
& =\operatorname{var}\left[\frac{1}{N} \sum_{k=1}^{N} \chi_{k}\right]=\frac{1}{N^{2}} \sum_{k=1}^{N} \operatorname{var}\left[\chi_{k}\right]=\frac{1}{N} G(1-G)
\end{aligned}
$$

where shorthand notation $G$ is defined as

$$
G \equiv G\left(\sqrt{\sigma_{1}^{2}+s_{1}^{2}}, \sqrt{\sigma_{2}^{2}+s_{2}^{2}}, d_{1}, d_{2}, b_{1}, b_{2},\left\{\boldsymbol{r}_{j}, j=1,2, \cdots, M\right\}\right)
$$

The standard deviation of damage fraction is

$$
\operatorname{std}\left[q_{\text {damage }}(\text { area target, } M \text { weapons })\right]=\sqrt{\frac{1}{N} G(1-G)}
$$

While the average damage fraction tells us the average number of target elements killed out of total $N$ target elements, the standard deviation of damage fraction describes the fluctuations/uncertainty in the actual number of target elements killed in individual realizations. Large standard deviation means large swing in the actual number of target elements killed from one realization to another. 
Note that this expression for the standard deviation is valid only in the absence of dependent and independent errors, for which we have $\left(\sigma_{1}, \sigma_{2}, d_{1}, d_{2}\right)=(0,0,0,0)$. When either the independent errors or dependent error or both are present, the standard deviation of damage fraction is larger than the value predicted by applying Equations (11) and (12) with non-zero $\left(\sigma_{1}, \sigma_{2}, d_{1}, d_{2}\right)$. We demonstrate this behavior numerically in Figure 2 and Figure 3 . We consider the model problem in which a single shot is fired against an area target of $N=20$ normally distributed elements. Monte Carlo simulations are carried out with $10^{6}$ runs for each set of parameter values and in each of the two situations below (i.e. without or with firing error), yielding accurate numerical results to compare with theoretical predictions.

In Figure 2, there is no firing error; the damage fraction is affected by the radius of area target (the standard deviation of target elements distribution). In this case, both the predicted mean and the predicted standard deviation of damage fraction are valid. As a result, the accurate Monte Carlo simulations agree with the theoretical predictions.

In Figure 3, the radius of area target is fixed at $s_{1}=s_{2}=10$; the damage fraction is affected by the firing error (the total effect of dependent and independent errors; for a single shot, there is no need to distinguish dependent and independent errors). In this case, only the predicted mean of damage fraction is valid. The predicted standard deviation calculated by applying Equations (11) and (12) with non-zero $\left(\sigma_{1}, \sigma_{2}, d_{1}, d_{2}\right)$ is invalid since Equations (11) and (12) are derived based on the assumption of zero firing error. The right panel of
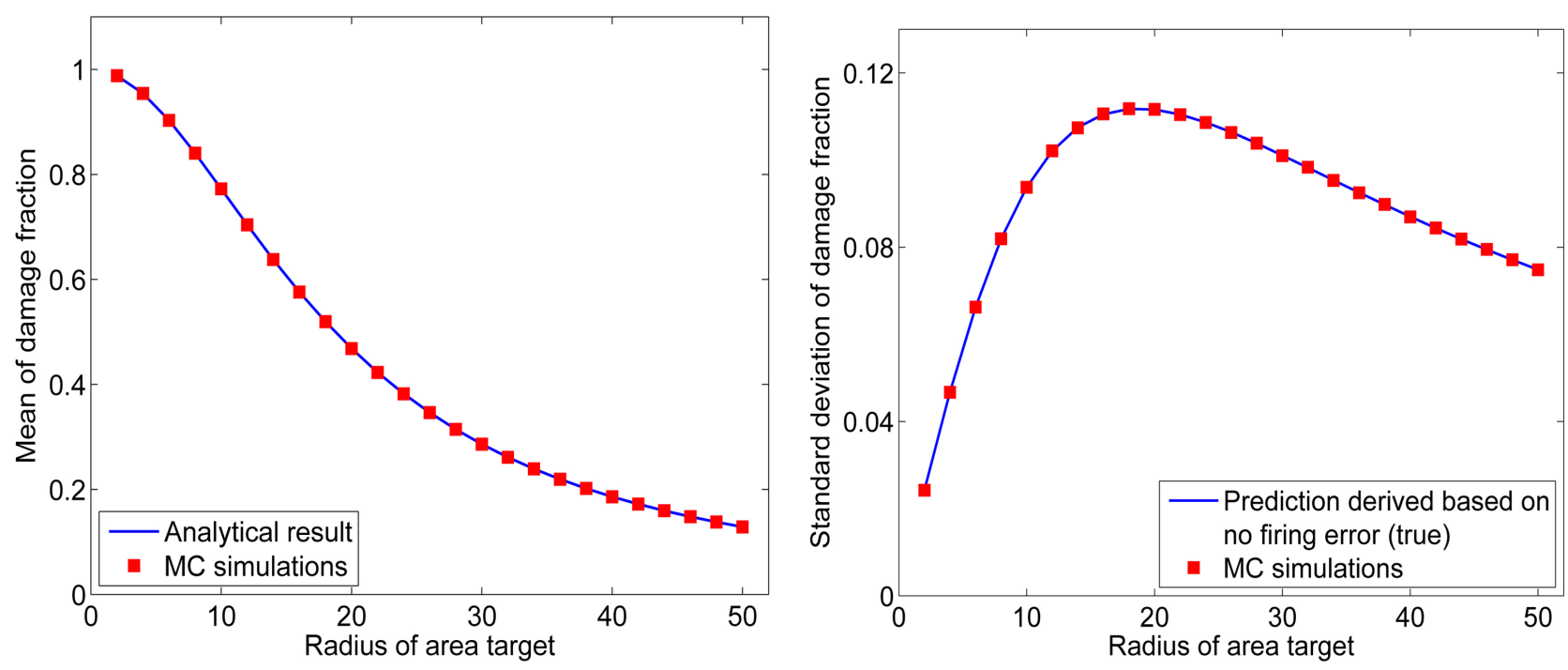

Figure 2. Statistics of the damage fraction for the case of a single shot against $N=20$ normally distributed target elements with no firing error $\left(\sigma_{1}, \sigma_{2}, d_{1}, d_{2}\right)=(0,0,0,0)$ and $\left(b_{1}, b_{2}\right)=(15,25)$. The radius of area target is defined as $s_{1}=s_{2}$ (the standard deviation of the elements distribution). Left panel: mean of damage fraction vs radius of area target. The analytical expression for the mean is valid regardless of the presence or absence of firing error. Right panel: standard deviation of damage fraction vs. radius of area target. The analytical expression for the standard deviation (Equations (11) and (12)) is valid only in the absence of firing error, which is true for the simulations in this figure. 

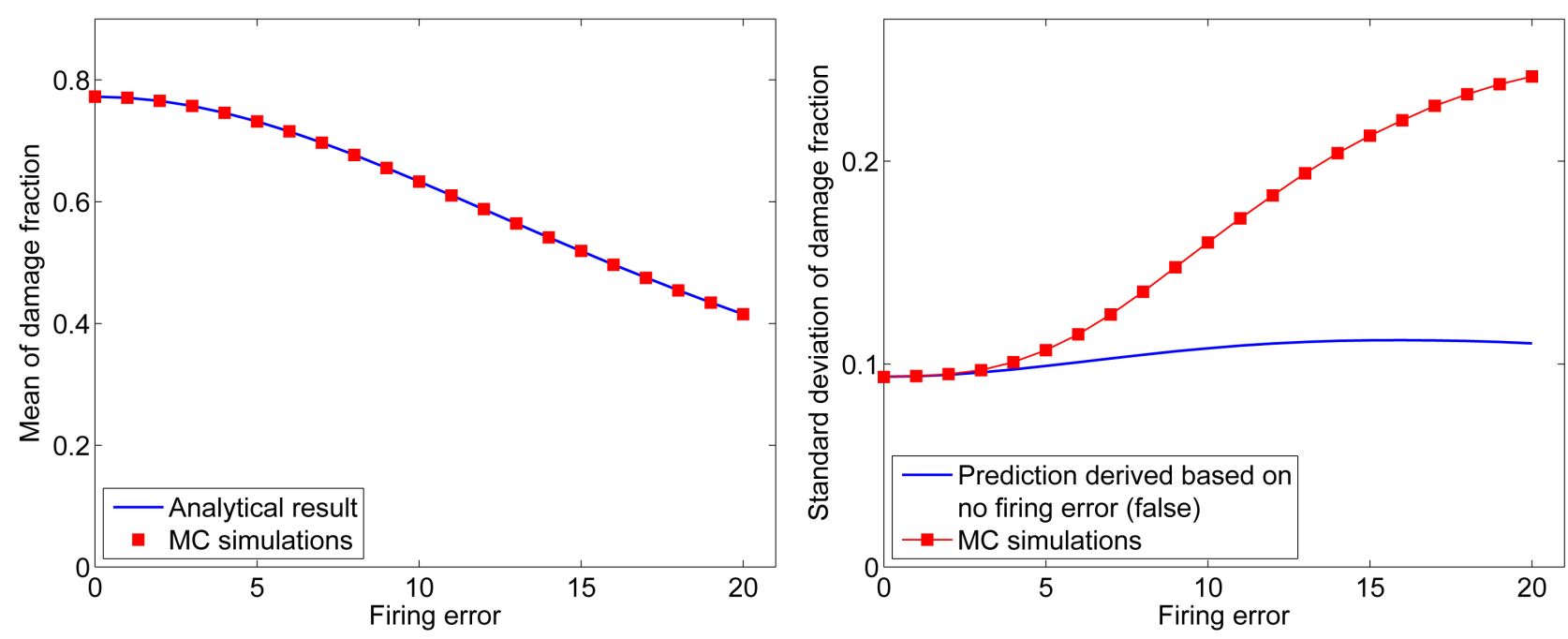

Figure 3. Statistics of the damage fraction for the case of a single shot against $N=20$ normally distributed target elements with $s_{1}=s_{2}=10, d_{1}=d_{2}=0$ and $\left(b_{1}, b_{2}\right)=(15,25)$. The firing error is defined as $\sigma_{1}=\sigma_{2}$. Left panel: mean of damage fraction vs firing error. The analytical expression for the mean is valid regardless of the presence or absence of firing error. Right panel: standard deviation of damage fraction vs firing error. The analytical expression for the standard deviation (Equations (11) and (12)) is valid only in the absence of firing error, which is false for the simulations in this figure. The results of Monte Carlo simulations show that in the presence of firing error, the actual standard deviation of the damage fraction is significantly larger than the value predicted by Equations (11) and (12) (which are not expected to be valid in the presence of firing error).

Figure 3 clearly demonstrates the deviation of the accurate Monte Carlo simulations from the invalid theoretical prediction.

\section{Optimal Aiming Points for an Area Target}

Next we investigate the optimal aiming points for the case of multiple weapons against an area target of normally distributed elements. We apply MATLAB built-in function "fminsearch" [6] on Formula (9) to find aiming points which produce the largest damage fraction. Technically MATLAB "fminsearch" yields only a local optimum. To find the global optimum, in each optimization we start MATLAB "fminsearch" with 5 random initial vectors. In all cases of our simulations, all 5 random initial vectors lead to the same optimum, indicating that the optimum found is very likely the global optimum.

In this study, in addition to finding the unconstrained overall optimal aiming positions, we also consider optimizations over a set of given constrained patterns of aiming points. The goal is to find simple and efficient "empirical" methods for calculating nearly optimal aiming positions. This approach greatly simplifies the numerical complexity of finding the optimal aiming points at the price of obtaining an approximate optimum. Based on our observations in simulations, computationally the overall optimization is at least two orders of magnitude (100 times) more expensive than the "empirical" optimization over a constrained pattern even when the significant additional cost of starting with multiple random vectors is excluded. In a sequential computing environment, starting the overall optimization with 5 random initial vectors makes the computation 5 
times expensive.

We consider 6 constrained patterns of aiming positions as listed below. These constrained patterns are motivated by the results of overall unconstrained optimization, some of which are shown in Figure 6, Figure 7 and Figure 10.

- Pattern A1: $M$ points on an ellipse, uniform in parameter angle.

Specifically, the $M$ aiming points are mathematically described by

$$
\begin{aligned}
& \theta_{j}=\theta_{1}+\frac{(j-1)}{M} 2 \pi, \quad j=1,2, \cdots, M \\
& \left(x_{j}, y_{j}\right)=R\left(\sqrt{\eta} \cos \theta_{j}, \frac{1}{\sqrt{\eta}} \sin \theta_{j}\right)
\end{aligned}
$$

This constrained pattern has three parameters: $\theta_{1}, R$ and $\eta$, over which we are going to optimize the average damage fraction. Here $\theta_{1}$ is the parameter angle for weapon $1 . R$ is the effective radius of the ellipse, satisfying

$$
R=\sqrt{\frac{\text { area of ellipse }}{\pi}}
$$

whereas $\eta$ is the aspect ratio of the ellipse, satisfying

$$
\eta=\frac{\text { major axis }}{\text { minor axis }}
$$

- Pattern A2: 1 point at center and $(M-1)$ points on an ellipse, uniform in parameter angle.

One aiming point is placed at the center. The rest $(M-1)$ aiming points are distributed along an ellipse, uniformly in parameter angle $\theta$, as described by Equations (13) and (14) where $M$ is replaced by $(M-1)$. This constrained pattern has three parameters: $\theta_{1}, R$ and $\eta$.

- Pattern A3: 2 points on the $x$-axis and $(M-2)$ points on an ellipse, uniform in parameter angle.

Two aiming points are placed, respectively, at $\left(x_{a}, 0\right)$ and $\left(-x_{a}, 0\right)$. The rest $(M-2)$ aiming points are distributed along an ellipse, uniformly in parameter angle $\theta$, as described by Equations (13) and (14) where $M$ is replaced by $(M-2)$. This constrained pattern has 4 parameters: $\theta_{1}, R, \eta$, and $x_{a}$.

- Pattern B1: $M$ points on an ellipse, uniform in polar angle.

The $M$ aiming points are mathematically described as follows. Their polar angles $\phi_{j}$ are given by

$$
\phi_{j}=\phi_{1}+\frac{(j-1)}{M} 2 \pi, \quad j=1,2, \cdots, M
$$

Their parameter angles $\theta_{j}$ are determined by the condition that the two vectors

$$
\left(\sqrt{\eta} \cos \theta_{j}, \frac{1}{\sqrt{\eta}} \sin \theta_{j}\right) \text { and }\left(\cos \phi_{j}, \sin \phi_{j}\right) \text { point in the same direction }
$$

Consequently, the aiming points on the ellipse can be described as 


$$
\left(x_{j}, y_{j}\right)=R\left(\sqrt{\eta} \cos \theta_{j}, \frac{1}{\sqrt{\eta}} \sin \theta_{j}\right)
$$

This constrained pattern contains three parameters: $\phi_{1}, R$ and $\eta$.

- Pattern B2: 1 point at center and $(M-1)$ points on an ellipse, uniform in polar angle.

One aiming point is placed at the center. The rest $(M-1)$ aiming points are distributed along an ellipse, uniformly in polar angle $\phi$, as described by Equations (15), (16) and (17) where $M$ is replaced by $(M-1)$. This constrained pattern has three parameters: $\phi_{1}, R$ and $\eta$.

- Pattern B3: 2 points on the $x$-axis and $(M-2)$ points on an ellipse, uniform in polar angle.

Two aiming points are placed, respectively, at $\left(x_{a}, 0\right)$ and $\left(-x_{a}, 0\right)$. The rest $(M-2)$ aiming points are distributed along an ellipse, uniformly in polar angle $\phi$, as described by Equations (15), (16) and (17) where $M$ is replaced by $(M-2)$. This constrained pattern includes four parameters: $\phi_{1}, R, \eta$, and $X_{a}$.

An example of Pattern B3 (2 points on the $x$-axis and rest of points on an ellipse, uniform in polar angle) is shown in the left panel of Figure 6. Pattern B1 (all points on an ellipse, uniform in polar angle) and Pattern B2 (1 point at center and rest of points on an ellipse, uniform in polar angle) are illustrated respectively in the left panel and in the right panel of Figure 7.

In numerical simulations below, we choose the parameter values as follows:

$M=1$ to 12 , number of weapons;

$\left(b_{1}, b_{2}\right)=(60,100)$, parameters in Carleton damage function;

$\left(\sigma_{1}, \sigma_{2}\right)=(5,5)$, standard deviation (s.d.) of dependent error in firing errors;

$\left(d_{1}, d_{2}\right)=(5,5)$, s.d. of independent errors in firing errors;

$s=s_{1}=s_{2}=15$ to 300 , radius of area target (s.d. of elements distribution).

We compare the results of optimization over constrained patterns with those of the overall optimization. Figure 4 plots the difference in optimal (maximum) damage fraction $\left(p_{\text {opt }}\right)$ between constrained and unconstrained optimizations as a function of area target radius $(s)$ for various numbers of weapons $(M)$. For small $s$, the difference in damage fraction is small among various constraints. This is intuitive and reasonable since for small $s$, the fatal area of each weapon is capable of covering the whole area target. For moderate to large $s$, the difference in damage fraction compares the performance of optimization constrained over each given pattern. For $M \leq 6$ (Figure 4(a)), the best approximate optimal damage fraction is achieved by distribute aiming points over an ellipse, uniformly in polar angle (Pattern B1 described above). At $M=7$ (Figure 4(b)), the best approximate optimal damage fraction is achieved by placing an aiming point at center and placing the rest six aiming points over an ellipse, uniformly in polar angle (Pattern B2). This is also true for $M=8$ and $M=9$. As the number of weapons increases, at $M=10$ (Figure $4(\mathrm{c})$ ), the best approximate optimal damage fraction is achieved by placing two aiming points on the $x$-axis 


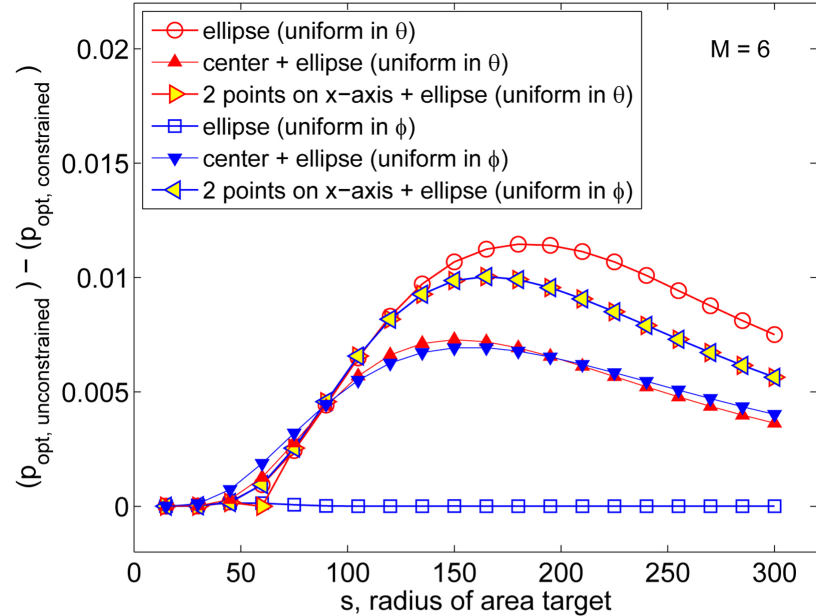

(a)

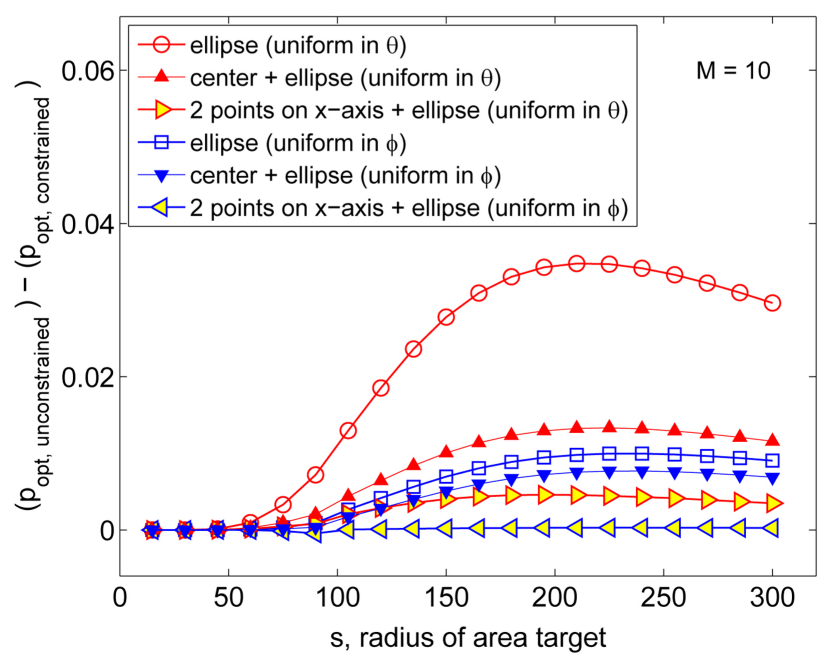

(c)

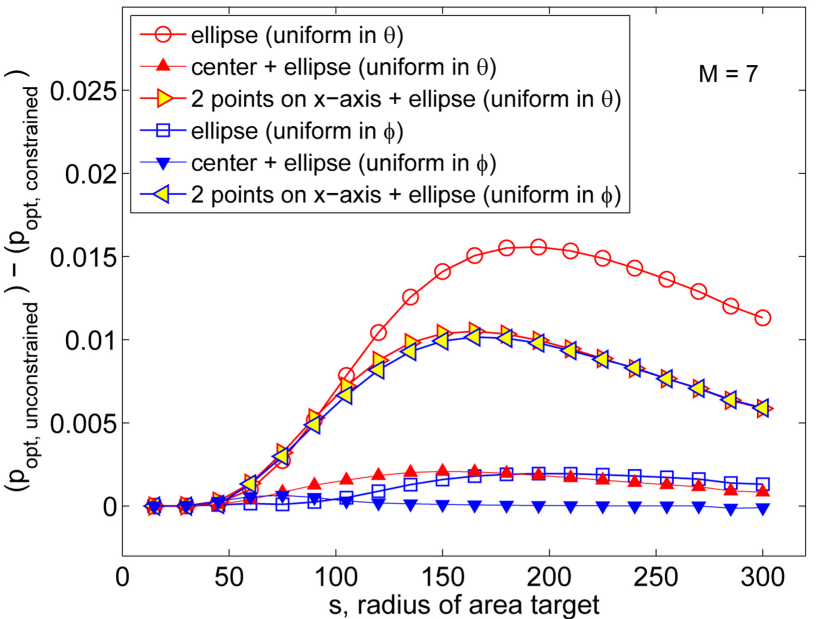

(b)

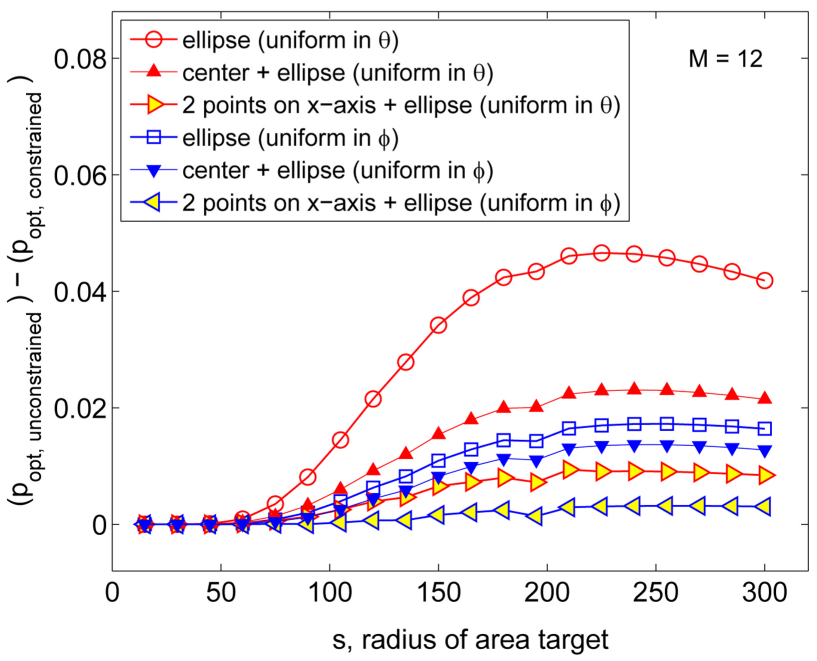

(d)

Figure 4. Difference in optimal damage fraction $\left(p_{\text {opt }}\right)$ between constrained and unconstrained optimizations as a function of area target radius $(s)$ for various numbers of weapons $(M)$. (a) $M=6$; (b) $M=7$; (c) $M=10$; (d) $M=12$.

and the rest eight aiming points over an ellipse, uniformly in polar angle (Pattern B3). The constrained optimum over Pattern B3 remains very accurate at $M=12$ weapons (Figure $4(\mathrm{~d})$ ).

In summary, as $M$ increases, the best pattern of aiming points for obtaining approximately the highest damage fraction goes from Patterns B1 to B2 to B3. This transition is clearly demonstrated in Figure 5 where the difference in optimal damage fraction $\left(p_{\text {opt }}\right.$ ) between constrained and unconstrained optimizations is shown as a function of $M$ at $s=150$ (radius of area target).

Figure 6 compares the unconstrained optimal aiming points and the optimal aiming points constrained to Pattern B3, respectively for $M=10$ and $M=12$ at $s=150$. At $M=10$, the optimal aiming points of Pattern B3 match almost exactly the unconstrained aiming points. At $M=12$, the optimal aiming points of Pattern B3 deviate from the unconstrained aiming points. Despite the apparent 


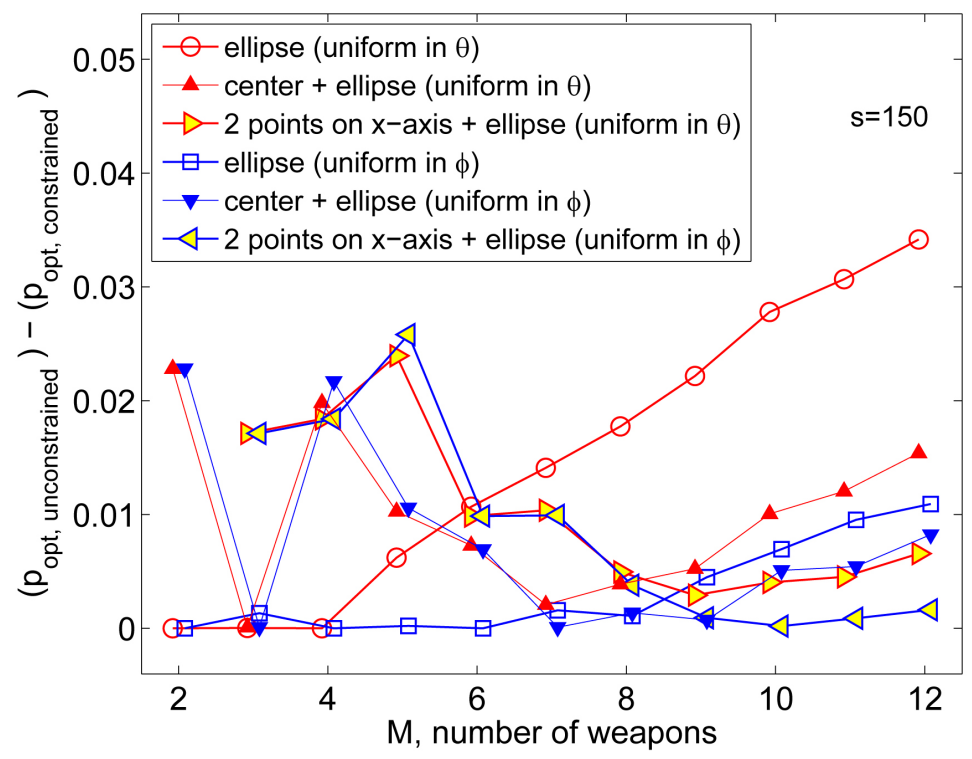

Figure 5. Difference in optimal damage fraction $\left(p_{\text {opt }}\right)$ between constrained and unconstrained optimizations as a function of $M$ at $s=150$ (radius of area target). In the horizontal direction, all points should have integer values. To visually display points that are on top of each other, they are shifted slightly in the horizontal direction in the plot.
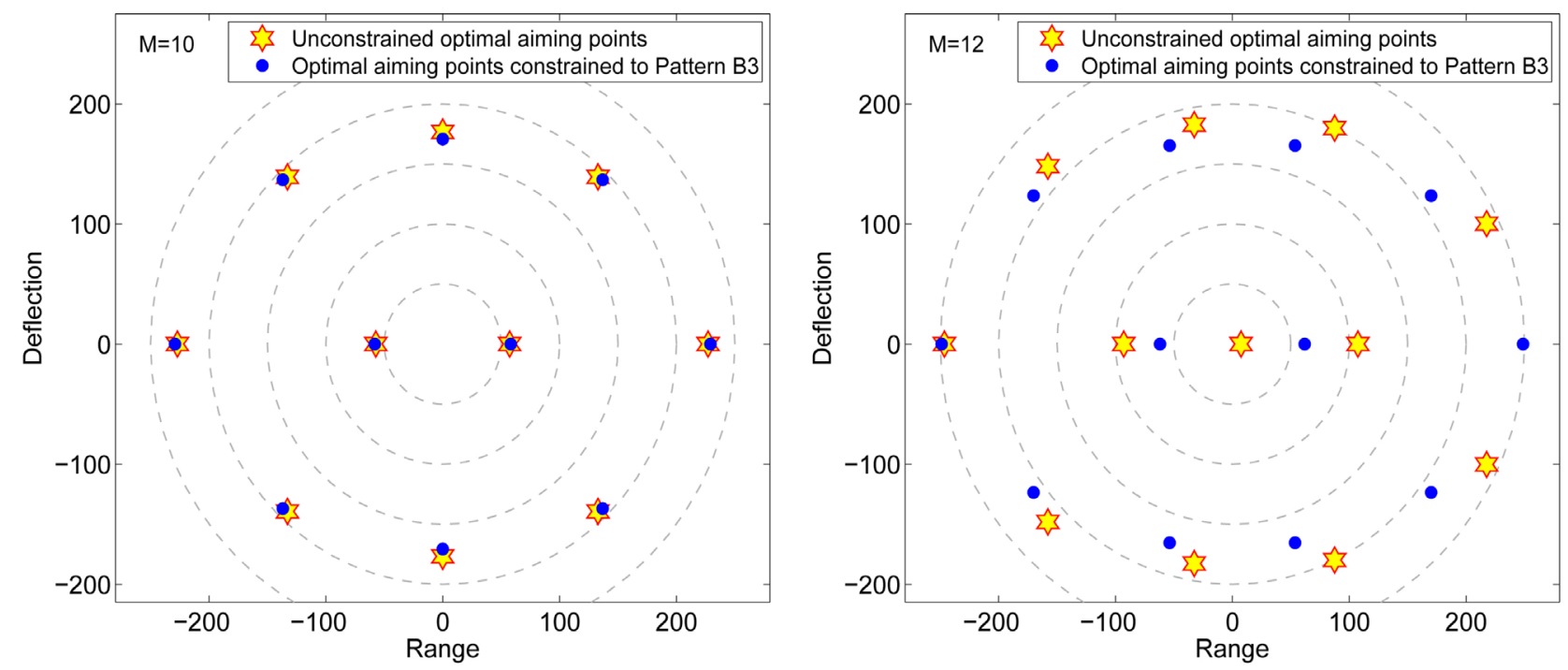

Figure 6. Comparison of unconstrained optimal aiming points and optimal aiming points constrained to Pattern B3. Left panel: two sets of optimal aiming points for $M=10$, yielding $p_{\{\text {opt,unconstrained }\}}=0.772604$ and $p_{\{\text {opt,Pattem B } 3\}}=0.772398$, respectively. Right panel: two sets of optimal aiming points for $M=12$, yielding $p_{\{\text {opt, unconstrained }\}}=0.814997$ and $p_{\{\text {opt,Pattern B3\} }}=0.813372$, respectively.

discrepancy between these two sets of optimal aiming points, the corresponding damage fractions are still very close to each other: the optimal damage fraction for Pattern B3 is $p_{\{\text {opt,Pattern B3\} }}=0.813372$ while the overall optimal damage fraction is $p_{\{\text {opt,unconstrained }\}}=0.814997$. The difference between these two damage fraction values is less than $0.2 \%$. It is important to point out the difference in 
computational complexity between these two optimizations. While the constrained optimization over Pattern B3 has 4 variables, the unconstrained optimization for $M=12$ weapons has 24 variables, which converges much slower than the constrained optimization.

The optimal aiming points constrained to Pattern $\mathrm{B} 1$ for $M=6$, the optimal aiming points constrained to Pattern B2 for $M=7$ and the corresponding unconstrained optimal aiming points are displayed in Figure 7.

Figure 8 plots the optimal damage fraction, respectively, as a function of $M$ for several values of $s$ (left panel), and as a function of $s$ for several values of $M$ (right panel). For a fixed value of $s$, the optimal damage fraction increases with
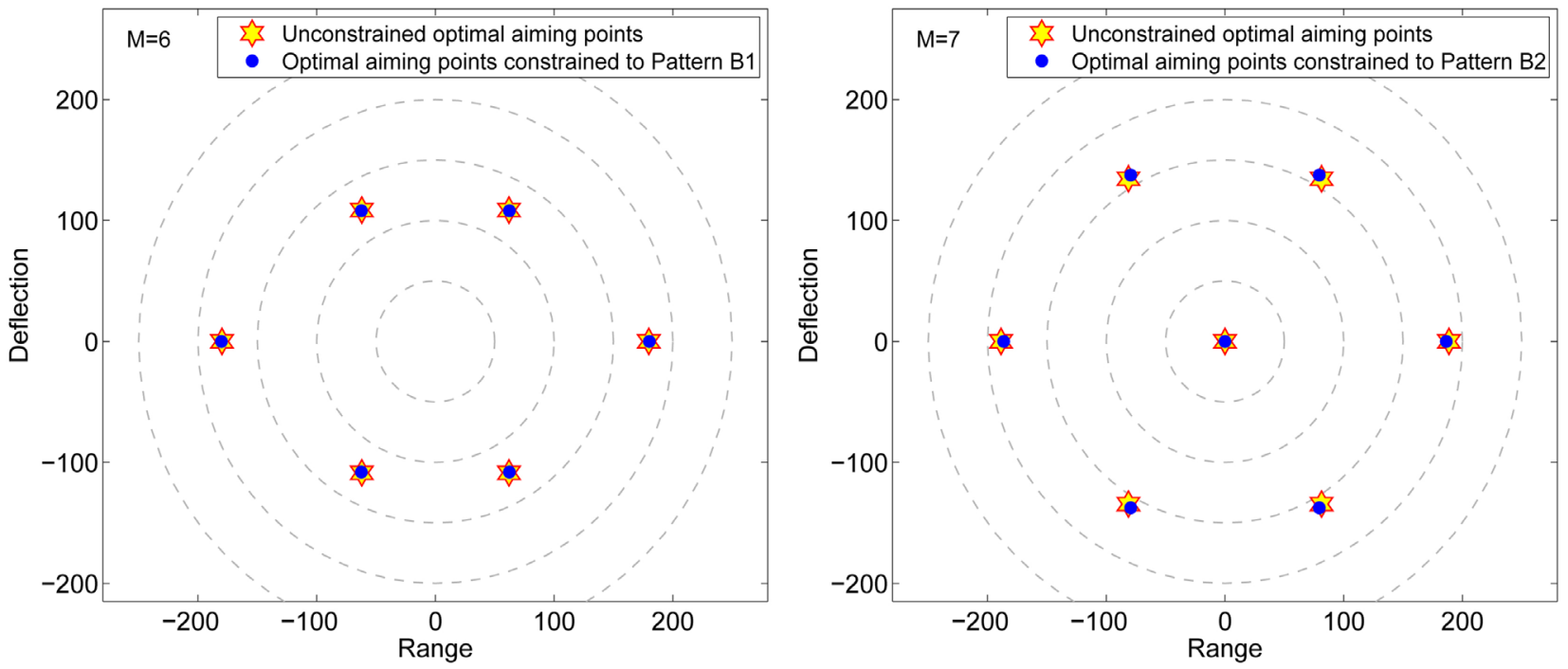

Figure 7. Left panel: unconstrained optimal aiming points and optimal aiming points constrained to Pattern $\mathrm{B} 1$ for $M=6$ weapons. Right panel: unconstrained optimal aiming points and optimal aiming points constrained to Pattern B2 for $M=7$ weapons.
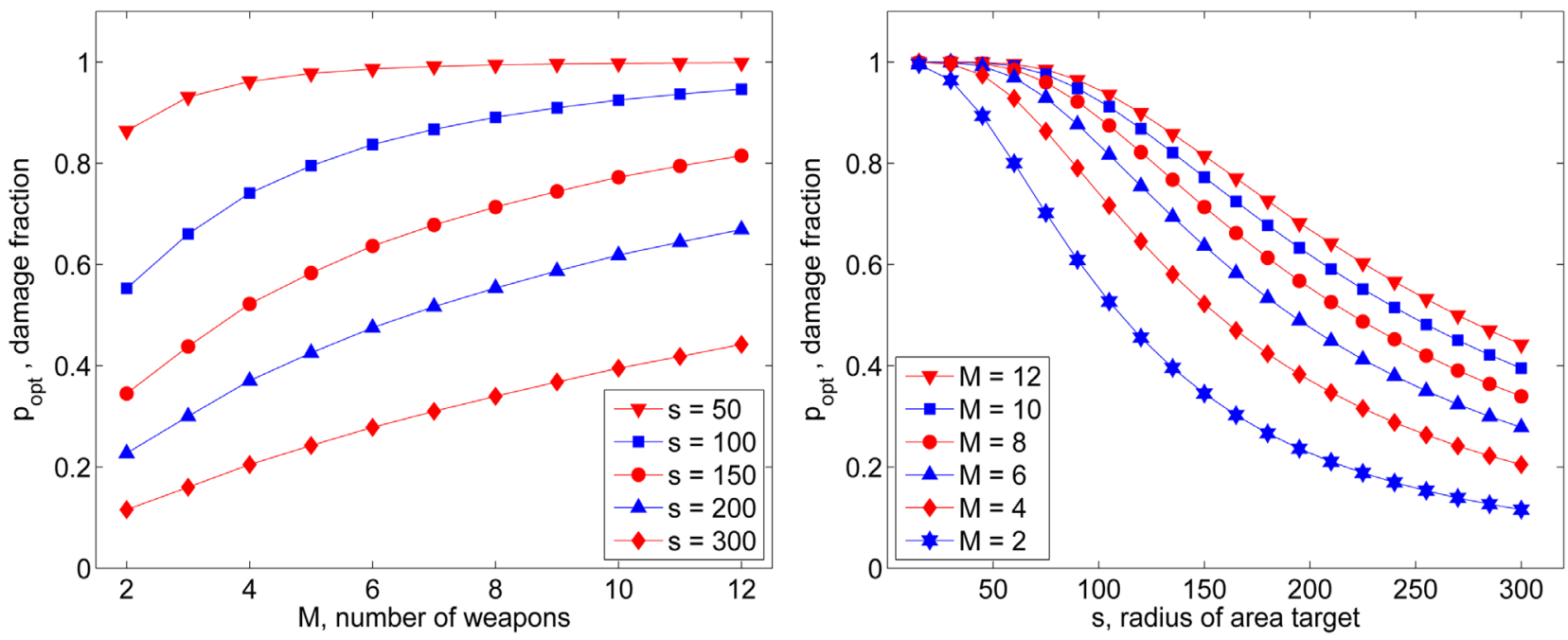

Figure 8. Damage fraction corresponding to the optimal aiming points. Left panel: damage fraction as a function of $M$. Right panel: damage fraction as a function of $s$. 
the number of weapons, $M$; for a fixed value of $M$, the optimal damage fraction decreases as the radius $(s)$ of area target is increased (i.e., damage fraction is lower for a larger area target). Both of these results are reasonable and consistent with our intuition.

A practical question regarding resource allocation is the following: Given the radius of area target $(s)$, what is the minimum number of weapons needed to achieve a given threshold of damage fraction? This question is answered in Figure 9. Figure 9 shows that for any given threshold of damage fraction, the minimum number of weapons needed is an increasing function of the area target radius (i.e., larger area target requires larger number of weapons), which again is reasonable and consistent with our intuition.

\section{Scaling Laws for Optimal Aiming Points and Optimal Damage Fraction with Respect to Area Target Radius}

Finally, we study how the optimal aiming points change with $s$, the radius of area target, and explore if there is a scaling law relating sets of optimal aiming points at different values of $s$. We start by examining the optimal aiming points for 4 different values of area target radius. The 4 panels in Figure 10 show the optimal aiming points for $M=10$ weapons, respectively, for $s=100, s=200, s=300$ and $s=400$. The spreading size of optimal aiming points increases as the radius of area target $(s)$ is increased. However, the increase does not follow a simple proportional linear relationship. Figure 10 indicates that the increase in the spread size of optimal aiming points is less than linear with respect to the area target radius. This can be explained intuitively as follows. When the radius

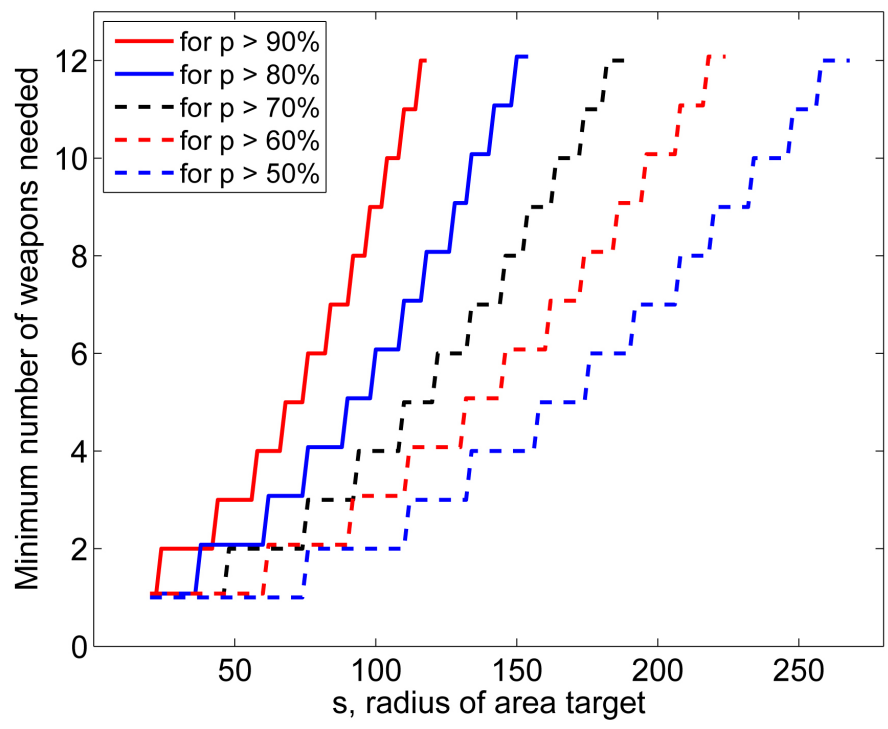

Figure 9. Minimum number of weapons needed for achieving a given threshold of damage fraction vs. radius of area target. In the vertical direction, all flat steps should have integer values. To visually display steps from different curves that are overlap with each other, they are shifted slightly in the vertical direction in the plot. 


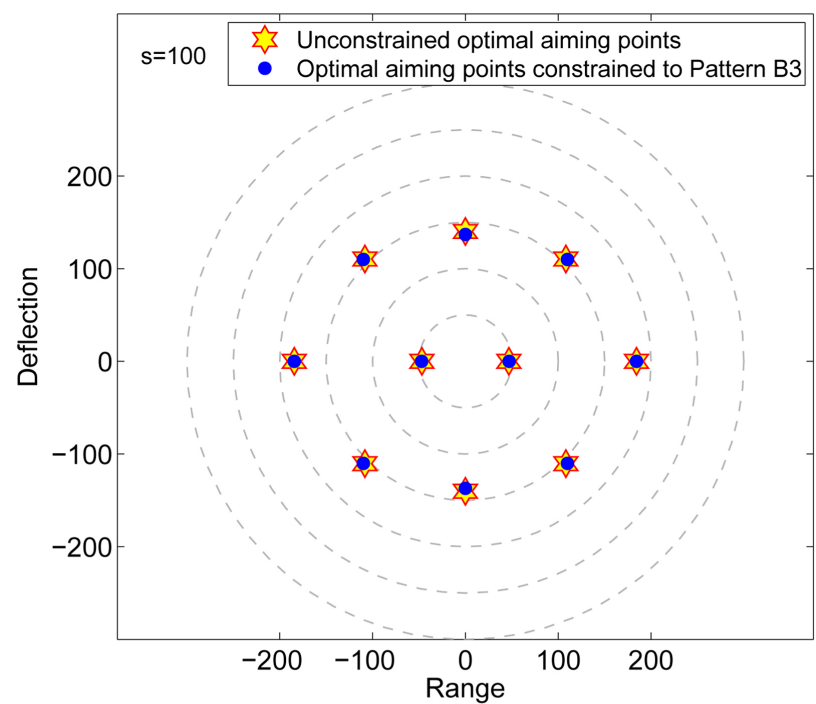

(a)

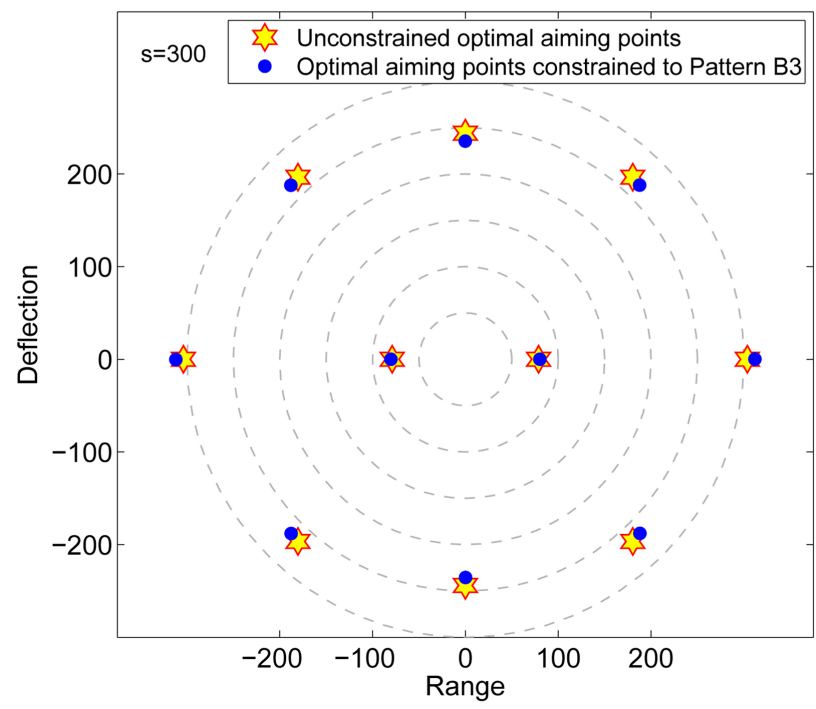

(c)

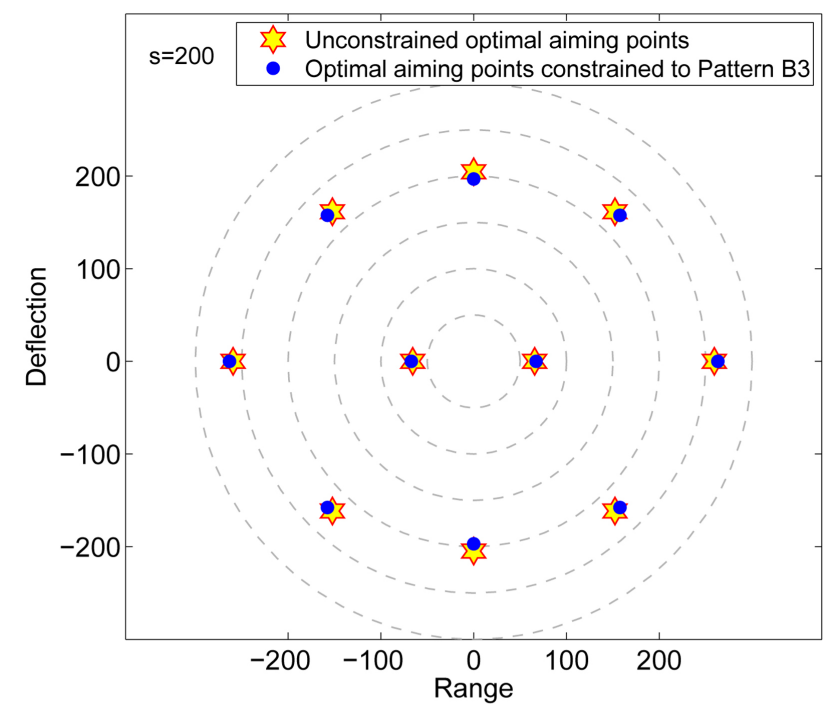

(b)

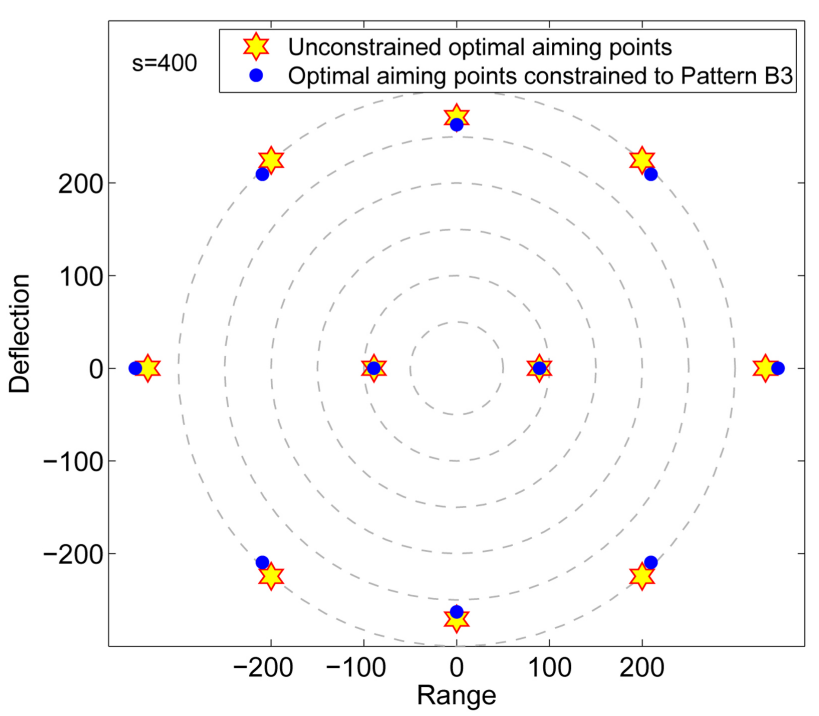

(d)

Figure 10. Sets of optimal aiming points for $M=10$ weapons for several values of area target radius $(s)$. (a) $s=100 ;($ b) $s=200 ;$ (c) $s=300 ;$ (d) $s=400$.

of area target is increased, the set of aiming points needs to cover a larger region. On the other hand, to maximize the damage fraction, the killing areas associated with individual weapons also need to maintain a certain degree of overlapping with each other. These two needs contradict each other and cannot be both accommodated simultaneously with a fixed number of weapons $(M)$ as the area target radius is increased. Thus, it is expected that as the radius of area target is increased, the spread size of optimal aiming points will increase less than linearly. Here we avoid using the term "radius of optimal aiming points" because the distribution of aiming points is not circularly symmetric.

For the purpose of investigating the spread size of aiming points quantitatively, 
we define the size of a set of $M$ aiming points $\left\{\boldsymbol{r}_{j}, j=1,2, \cdots, M\right\}$ mathematically as

$$
L_{\mathrm{AP}} \equiv \sqrt{\frac{1}{M} \sum_{j=1}^{M}\left|\boldsymbol{r}_{j}\right|^{2}}
$$

To explore how the size of optimal aiming points scales with the area target radius, we plot these two quantities against each other in a log-log plot in the left panel of Figure 11, which also includes a fitting function of the form $L_{\mathrm{AP}} \propto \sqrt{s}$. The log-log plot along the fitting function indicates that the size of optimal aiming points $\left(L_{\mathrm{AP}}\right)$ approximately is proportional to the square root of area target radius $(\sqrt{s})$. These simulation results lead us to the empirical conclusion that the size of optimal aiming point distribution scales as the square root of area target radius. Based on this key observation, we introduce the scaled aiming points as

$$
\boldsymbol{r}_{j}^{(\text {scaled })}(s) \equiv \frac{1}{\sqrt{s}} \boldsymbol{r}_{j}(s)
$$

The right panel of Figure 11 compares four sets of scaled optimal aiming points of Pattern B3 for $s=100, s=200, s=300$ and $s=400$, respectively. The comparison demonstrates that not only the spread size of optimal aiming points scales as $\sqrt{s}$, the distribution of optimal aiming points after scaling is approximately invariant with respect to the area target radius. Mathematically, we have observed that approximately

$$
\boldsymbol{r}_{j}^{\text {(scaled) }}(s) \text { is invariant with respect to } s
$$

This scaling property gives us an even more efficient way of calculating optimal aiming points. We only need to calculate the optimal aiming points for
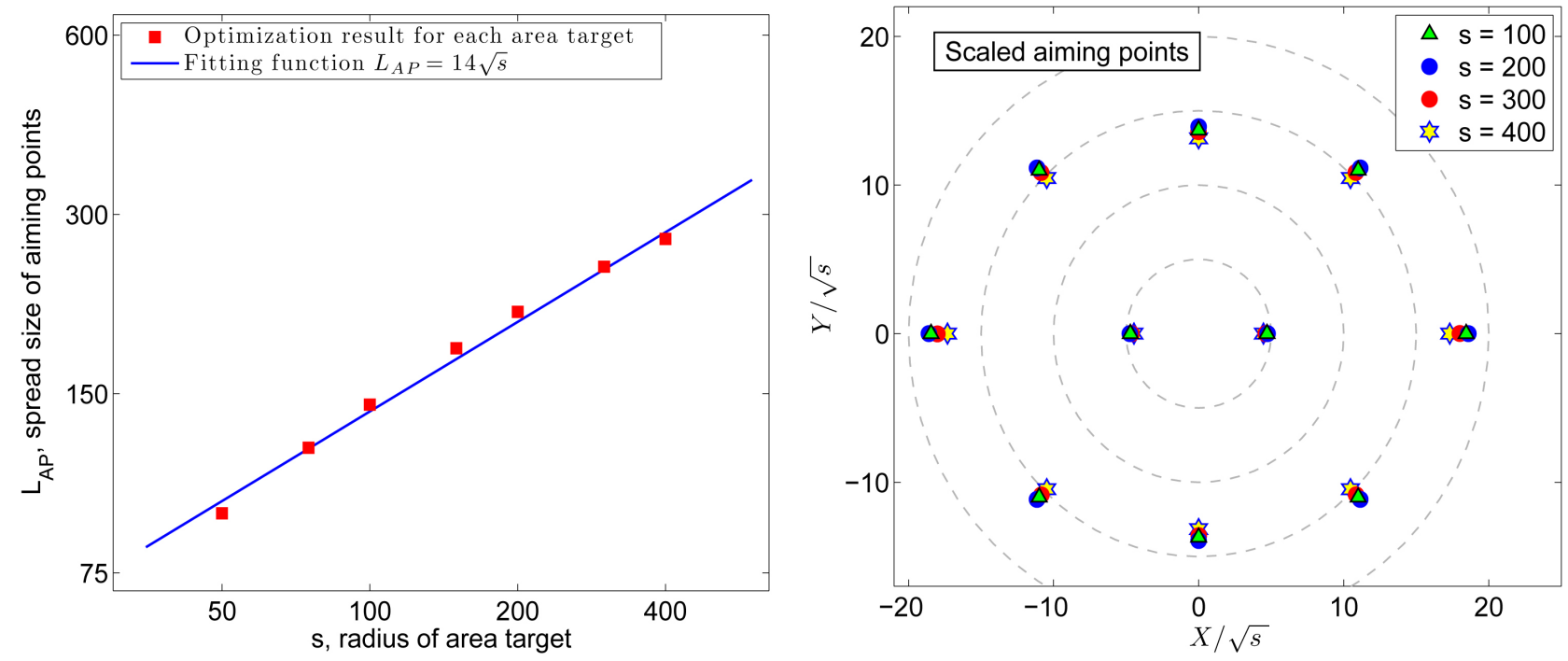

Figure 11. Comparison of optimal aiming points for different values of area target radius. Left panel: spread size of optimal aiming points vs. radius of area target in a log-log plot. Right panel: sets of scaled optimal aiming points of Pattern B3 for $s=100$, $s=200, s=300$ and $s=400$. 
an area target of typical/representative radius: $\left\{\boldsymbol{r}_{j}\left(s_{0}\right), j=1,2, \cdots, M\right\}$. We use $s_{0}=150$ in our study. For an area target of radius $s$, we simply calculate/predict a set of nearly optimal aiming points from $\left\{\boldsymbol{r}_{j}\left(s_{0}\right)\right\}$ using the scaling law.

$$
\boldsymbol{r}_{j}(s)=\sqrt{\frac{s}{s_{0}}} \boldsymbol{r}_{j}\left(s_{0}\right)
$$

We evaluate the performance of this efficient method by examining the damage fraction values achieved by these sets of nearly optimal aiming points. Specifically, for each area target, we calculate the damage fraction values corresponding respectively to three sets of aiming points:

- aiming points calculated in the unconstrained optimization;

- aiming points calculated using scaling law (21);

- all aiming points $=(0,0)$.

Figure 12 compares the damage fraction values caused by the 3 sets of aiming points described above for $M=6$ weapons (left panel) and for $M=10$ weapons (right panel). The damage fraction achieved by the set of nearly optimal aiming points calculated using scaling is indistinguishable from that achieved in the unconstrained optimization (true optimum) while the damage fraction corresponding to all weapons aiming at $(0,0)$ is much lower. Therefore, we conclude that scaling law (21) is an efficient and accurate method for calculating a set of nearly optimal aiming points.

\section{Concluding Remarks}

We have studied the average damage fraction of an area target caused by multiple weapons. The area target was assumed to consist of normally distributed elements. Using the analytical expression of the average damage fraction, we compared various distribution patterns of the aimpoints and gave optimal
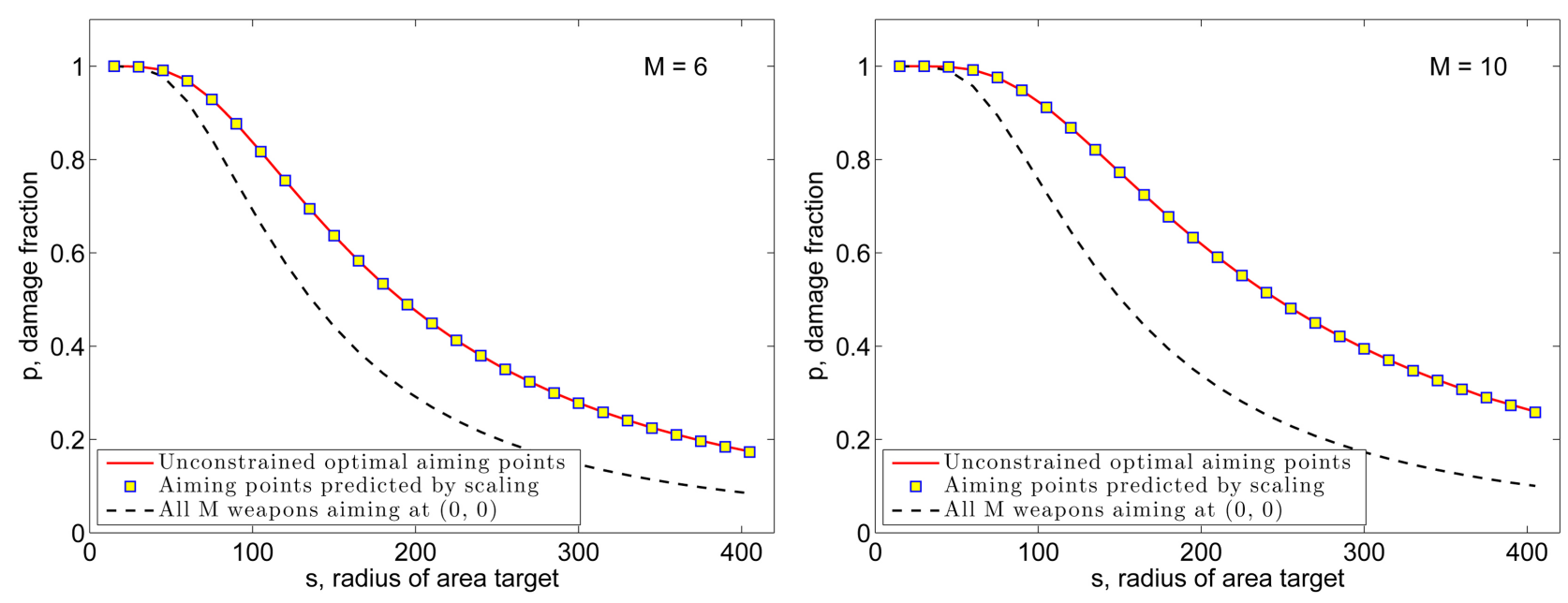

Figure 12. Comparison in damage fraction performance of 3 sets of aiming points: 1) aiming points calculated in the unconstrained optimization; 2) aiming points calculated using scaling; and 3) all aiming points $=(0,0)$. Left panel: $M=6$ weapons. Right panel: $M=12$ weapons. 
patterns for different number of weapons. Scaling laws for optimal aimpoints and optimal damage fraction with respect to the radius of the area target were derived. One prospective future research is to extend our current work to an area target of uniformly distributed elements. Another avenue for future research is to consider an area target where the elements are assigned different values and seek optimal aimpoints in order to minimize the total average surviving value.

\section{Disclaimer}

The authors would like to thank TRAC-Monterey for supporting this work and Center for Army Analysis (CAA) for bringing this problem to our attention. The views expressed in this document are those of the authors and do not reflect the official policy or position of the Department of Defense or the U.S. Government.

\section{References}

[1] Eckler, A. and Burr, S. (1972) Mathematical Models of Target Coverage and Missile Allocation. Military Operations Research Society, Alexandria, VA.

[2] Washburn, A. and Kress, M. (2009) Combat Modeling. Springer, New York, NY. https://doi.org/10.1007/978-1-4419-0790-5

[3] Driels, M. (2014) Weaponeering: Conventional Weapon System Effectiveness. 2nd Edition, American Institute of Aeronautics and Astronautics (AIAA) Education Series, Reston, VA.

[4] Washburn, A. (2013) Diffuse Gaussian Multiple-Shot Patterns. Military Operations Research, 8, 59-64

[5] Wang, H., Moten, C., Driels, M., Drundel, D. and Zhou, H. (2016) Explicit Exact Solution of Damage Probability for Multiple Weapons against a Unitary Target. American Journal of Operations Research, 6, 450-467. https://doi.org/10.4236/ajor.2016.66042

[6] Lagarias, J.C., Reeds, J.A., Wright, M.H. and Wright, P.E. (1998) Convergence Properties of the Nelder-Mead simplex Method in Low Dimensions. SIAM Journal of Optimization, 9, 112-147. https://doi.org/10.1137/S1052623496303470 
Submit or recommend next manuscript to SCIRP and we will provide best service for you:

Accepting pre-submission inquiries through Email, Facebook, LinkedIn, Twitter, etc. A wide selection of journals (inclusive of 9 subjects, more than 200 journals)

Providing 24-hour high-quality service

User-friendly online submission system

Fair and swift peer-review system

Efficient typesetting and proofreading procedure

Display of the result of downloads and visits, as well as the number of cited articles Maximum dissemination of your research work

Submit your manuscript at: http://papersubmission.scirp.org/

Or contact ajor@scirp.org 